\title{
Turbulence and Bubble Break up in Slug Flow with Wall Injection
}

\author{
Francisco J. S. Bandeira' ${ }^{1}$ Gabriel F. N. Gonçalves ${ }^{1}$. \\ Juliana B. R. Loureiro ${ }^{1}$ Atila P. Silva Freire ${ }^{1}$
}

Received: 23 December 2015 / Accepted: 17 October 2016 / Published online: 28 October 2016

C) Springer Science+Business Media Dordrecht 2016

\begin{abstract}
The present work investigates experimentally the changes on the properties of horizontal slug flows subject to fluid injection at the wall. Measurements include data on global flow rates, pressure drop and local mean and fluctuating velocity profiles for nine different conditions. The properties of the two-phase flow are measured through a Shadow Sizer system and laser-based sensors. Two distinct flow transpiration rates are studied, $v_{w i}^{++}=v_{w} / U_{m}=0.0005$ and 0.001 . The effects of flow transpiration were observed to induce bubble break-up and large changes in the passage frequency and characteristic lengths of the unit cells. In addition to the two-phase flow results, single-phase flow measurements are presented with a view to compare the different turbulent effects introduced by the second phase. The work also proposes modifications in the models of Dukler and Hubbard (Ind. Eng. Chem. Fund. 14 337-347 (1975)) and Orell (Chem. Eng. Sci. 60 1371-1381 (2005)) so that fluid injection at the wall can be accounted for. All theoretical predictions are compared with the experimental data.
\end{abstract}

Keywords Turbulence $\cdot$ Bubble break up · Slug flow $\cdot$ Wall transpiration · Law of resistance

\section{Introduction}

Turbulent flow through straight circular pipes is considered by Townsend [1] the most important class of basic flows bounded by rigid walls. Single phase flows limited by impermeable walls are the most symmetrical and homogeneous of all turbulent shear flows and

Juliana B. R. Loureiro

jbrloureiro@mecanica.coppe.ufrj.br

1 Programa de Engenharia Mecanica (COPPE/UFRJ), Universidade Federal do Rio de Janeiro, C.P. 68503, 21941-972, Rio de Janeiro, Brazil 
have the particular property that all mean values - except pressure - are dependent on one co-ordinate, the radial direction.

However, once a fluid is introduced uniformly over a porous surface, the details of the flow change significantly. The addition of mass at the wall implies that solutions must now incorporate not only the effects of friction but also of acceleration. Turbulent quantities are further altered by the injection of fluid, in a very complicate manner that has not been completely understood.

The flow complexities described above are immensely aggravated by the presence of a second flow phase, gas. In fact, and irrespective of the specified liquid and gas flow rates, this is a problem that has been deficiently addressed in literature. One particular research topic of great relevance is the description of slug flow subject to fluid transpiration at the wall.

The purpose of the present work is to carry out reference experiments in horizontal pipes with fluid injection at the wall for single- and two-phase (slug) flow patterns. Measurements of global flow rates and pressure drop are made for different injection rates. In addition, the work presents local mean and fluctuating turbulent profiles obtained through Laser-Doppler Anemometry. The properties of the two-phase flow are measured through a Shadow Sizer system and laser-based sensors. Results showing the effects of fluid injection at the wall on the translational velocity of unit cells, liquid film length and passage frequency of long bubbles are also presented. The validity of the law of resistance previously advanced by Loureiro and Silva Freire [2] for single phase flow is also investigated through the new data.

To account for the effects of fluid injection at the wall on slug flow, modifications in the unit cell models of Dukler and Hubbard [3] and Orell [4] are proposed. Unit cell models are of undisputed importance in the numerical prediction of industrial problems. The two models considered here are based on different modeling parameters. For example, the model of Dukler and Hubbard [3] (D\&H) considers the shape of the large bubbles and a shedding rate relation for the prediction of the front propagation velocity $\left(V_{t}\right)$. The model of Orell [4] considers cylindrical bubbles and uses an input relation for $V_{t}$.

In unit cell models, a typical cell is postulated to repeat itself moving down a pipe. Provided a reference frame exists where the liquid and gas phases are considered to travel in a fully developed state with about the same velocity, the flow randomness can be encapsulated in models with fixed or stochastic cell lengths (Fabre and Line [5]). In the present work, modifications to account for fluid injection at the wall include an extra acceleration term and changes in the friction coefficient in the liquid slug. Of course, the continuous and homogeneous injection of fluid at the wall implies that the unit cell changes properties locally, in a dynamic behavior that must the tracked by the numerical computations.

Important properties of slug flow including the frequency of unit cells $\left(v_{s}\right)$ and the length $\left(l_{f}\right)$ distribution of long bubbles have not been previously discussed in the literature in connection with wall transpiration. The flow acceleration provoked by the wall injection of fluid and the very high levels of turbulence observed in the near wall region are shown here to exert a large influence on both $v_{s}$ and $l_{f}$.

The theory of Loureiro and Silva Freire [2] advanced for the prediction of the skinfriction coefficient in transpired turbulent pipe flows is briefly reviewed here. The unit cell models of Dukler and Hubbard [3] and Orell [4] for slug flow are also introduced. This section also shows how both theories are merged to yield a theory for slug flow subject to wall transpiration.

The experiments are described in a separate section, after which validation of the theoretical predictions is carried out. 


\section{Preliminaries}

A discussion on the physics of the effects of fluid injection at the wall on water-air horizontal slug flow is presented next. The reconstruction of bubble shape was made according to the fixed window method described in Matamoros et al. [6].

In what follows, $v_{w i}^{++}=v_{w} / U_{m}$, where $v_{w}$ is the normal injection velocity at the wall and $U_{m}$ is the bulk velocity in the entrance section of the pipe.

The shape of a typical bubble at position $x=7 \mathrm{~m}$ of the test section of the present experimental apparatus and with no fluid injection at the wall is shown in Fig. 1. The liquid slug and liquid film are fully developed and relatively free of small bubbles. Experiments on the evolution of slug frequencies performed by Ujang et al. [7] indicate that for the range of liquid and gas flow rates used in the present work, fully developed slug frequencies are obtained for lengths over 75 pipe diameters. At position $x=7 \mathrm{~m}$, the slug development length was about 225 pipe diameters.

Fluid injection at the wall is considered next. In the measurement section located 13 meters downstream of the inlet (Figs. 2 and 3), the high levels of near wall turbulence break up the long bubbles and aerate the flow provoking the appearance of small bubbles in the whole extent of the unit cell. The perturbations caused by the fluid injection tend to decrease the mean size of the large bubbles and increase their frequency of passage.

For the lower injection rate, $v_{w i}^{++}=0.0005$, (where the subscript $i$ indicates that $U_{m}$ is considered at the inlet) the strong disturbances on the body of the large bubble are clearly visible (Fig. 2). The many detached small bubbles tend to migrate to the upper part of the pipe, forming a layer of bubbles underneath which the large bubble travels.

An increase in the injection rate $\left(v_{w i}^{++}=0.001\right)$ thickens the layer of dispersed small bubbles, forcing the large bubbles to occupy the lower half of the pipe (Fig. 3a). The intense bubble break up process eventually gives origin to a flow pattern characterized by large bubbles that are much reduced in size (Fig. $3 b-c)$ and very long and extremely aerated liquid slugs (Fig. 3d).

The flow pattern shown in Fig. 3 illustrates some of the difficulties that are commonly associated with the application of experimental techniques for the determination of the flow properties. The large number of closely packed small bubbles throughout the entire unit cell tends to distort the signal produced by a given sensor, introducing noise that is difficult to be interpreted.

Typical signals furnished by a laser phase detection sensor are shown in Fig. 4. At $x=$ $3 \mathrm{~m}$ from the pipe inlet, the shapes of the bubbles are still well discerned (Fig. 4a), so that their lengths and passage frequencies can be characterized. As the turbulence level increases downstream $(x=13 \mathrm{~m})$, the laser light is strongly scattered giving rise to a signal of difficult analysis (Fig. 4b).

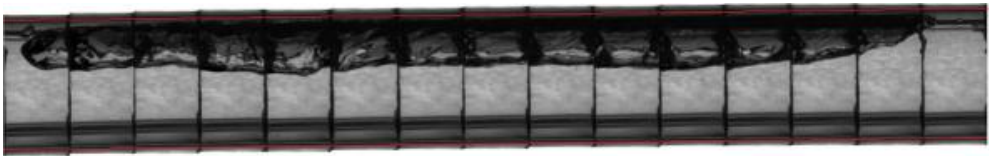

Fig. 1 Bubble shape at $x=7$ meters with no fluid injection at the wall $\left(v_{w i}^{++}=0\right) . Q_{l}=2.46 \mathrm{~m}^{3} \mathrm{~h}^{-1}, Q_{g}=$ $2.06 \mathrm{~m}^{3} \mathrm{~h}^{-1}$. The top and bottom limits of the pipe are shown in red 


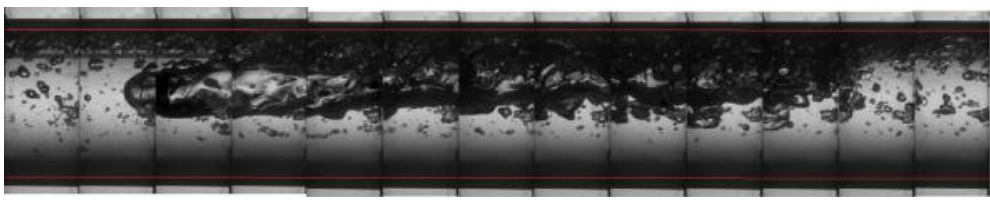

Fig. 2 Bubble shape at $x=7$ meters for $Q_{l}=2.46 \mathrm{~m}^{3} \mathrm{~h}^{-1}, Q_{g}=3.2 \mathrm{~m}^{3} \mathrm{~h}^{-1}$ and $v_{w i}^{++}=v_{w} / U_{m}=0.0005$, where $v_{w}$ is the normal velocity at the wall and $U_{m}$ is the bulk velocity in the entrance section of the pipe. The top and bottom limits of the pipe are shown in red

To determine some of the flow properties of interest the choice was then to use the Shadow Sizer Technique. Bubble lengths and slug frequencies were obtained directly from high speed photographs.

\section{Theoretical Background}

For laminar flow, the Navier-Stokes equations can be solved to unveil the dependence of the velocity components and pressure on position coordinates, pipe dimensions and fluid properties. Berman [8] proposed a classical solution for two-dimensional, incompressible, steady-flow in a channel. Other authors have examined Berman's solution to consider pressure-dependent wall suction [9] or the effect of slip boundary condition [10].

The above mentioned analyses consider symmetric flows in ducts with symmetric boundary conditions. Extension of the solutions to circular pipes were provided, e.g., by Erdogan and Imrak [11] and Tsangaris et al. [12].

For high Reynolds number flow, the search for analytical solutions is much complicated by the natural requirement of turbulence closure. Additionally, turbulent flow is known to be sensitive to wall roughness. For external flow, some authors have resorted (Stevenson

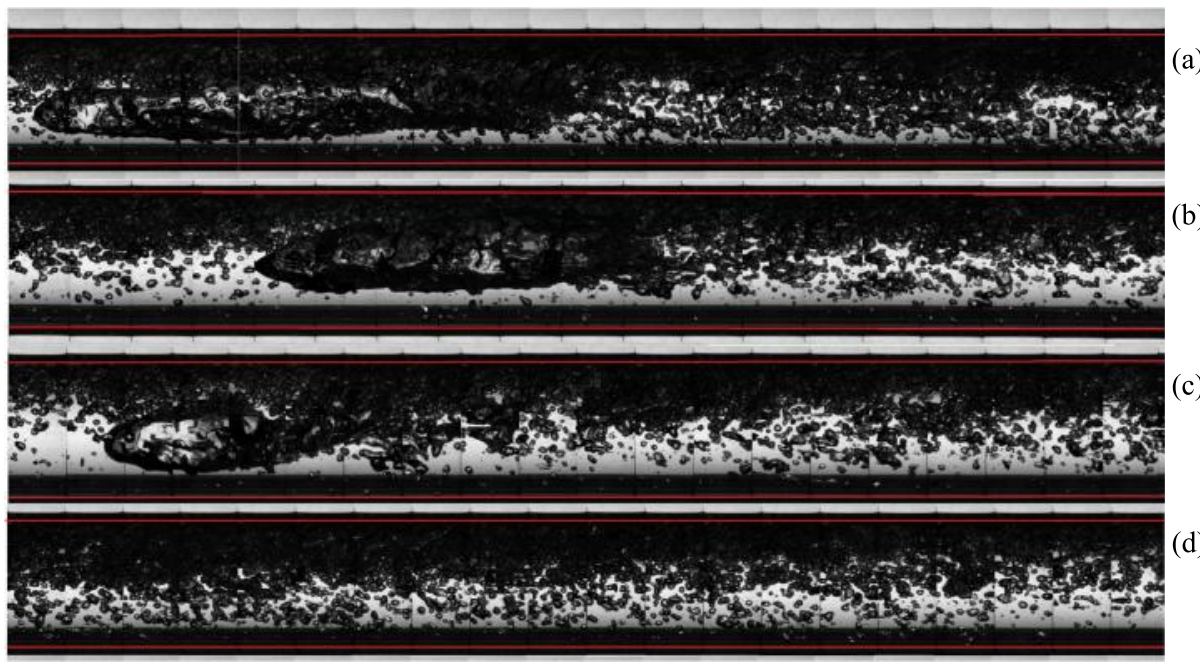

Fig. 3 Bubble shape at $x=13 \mathrm{~m}$ (a-b-c) and liquid slug pattern (d), for $Q_{l}=2.7 \mathrm{~m}^{3} \mathrm{~h}^{-1}, Q_{g}=2.6 \mathrm{~m}^{3} \mathrm{~h}^{-1}$ and $v_{w i}^{++}=0.001$. The top and bottom limits of the pipe are shown in red 

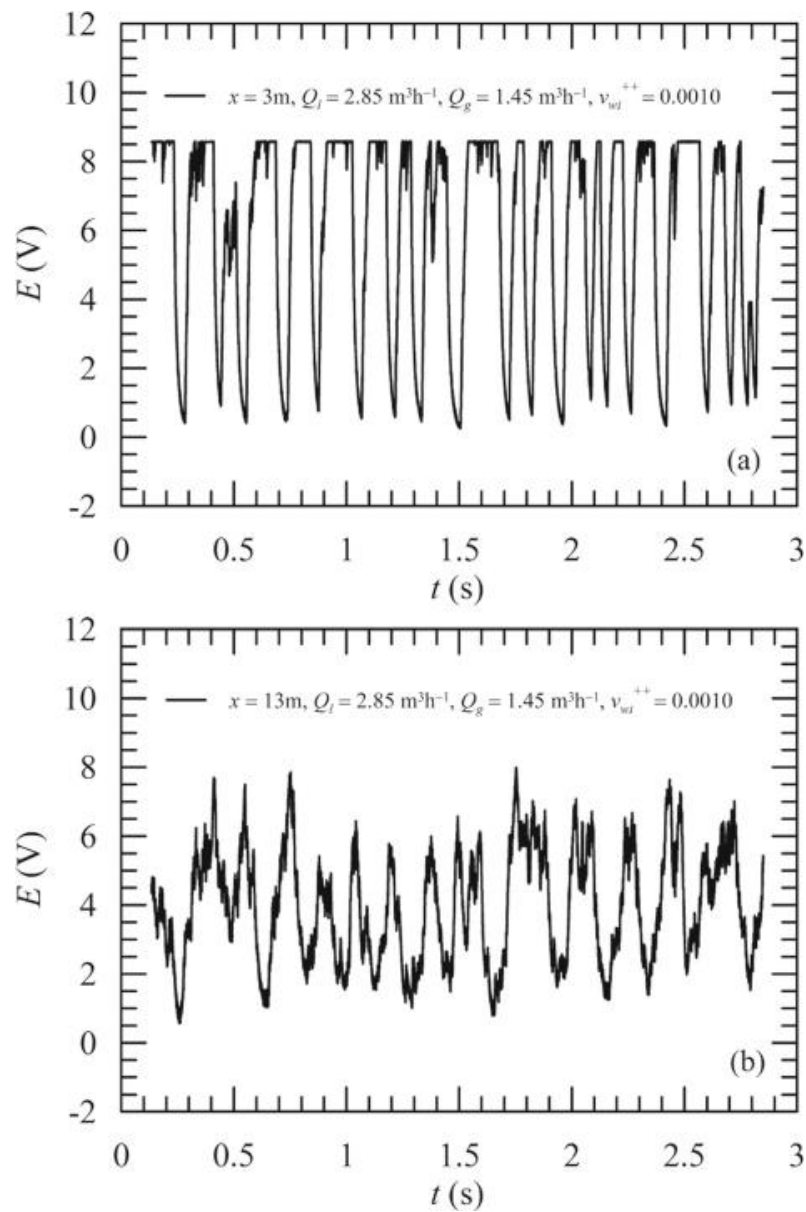

Fig. 4 Typical signals of the phase detection sensor. The highest values of $E$ correspond to the liquid phase

[13], Simpson [14], Silva Freire [15]) to simple algebraic closure hypotheses and perturbation arguments to develop local analytical solutions for the fully turbulent region. These solutions exhibit bi-logarithmic terms and incorporate the effects of local Reynolds number and transpiration rate. One prominent feature of the resulting solution is the dependence of an integration parameter on the transpiration rate.

The underlying assumption of the above mentioned analytical methods is that flow transpiration at the wall is homogeneous. Some applications, however, need to be approached differently. They consider fluid injection through isolated perforations, so that frictional losses need to be described through a decomposition of effects: wall friction and mixing effects. The latter effect is compared to the problem of multiple interacting jets in a cross flow. This approach results in a very intricate analysis so that only external empirical evidence can be used to determine the correct behavior of the pressure loss. Fortunately, in many applications the injection of fluid over the wall is to a good approximation uniform. Typical examples include the thermal protection of walls, filtration or the production of oil in horizontal or vertical wells. 
The law of resistance introduced in Loureiro and Silva Freire [2] is valid for single-phase flow and incorporates the effects of roughness and wall transpiration. The law is derived from a perturbation solution (see also Silva Freire [15]); a short presentation of the main results follows next.

\subsection{Resistance law for single phase flow in smooth pipes}

The friction coefficient $f$ can be defined through

$$
\frac{p_{1}-p_{2}}{L}=\frac{f}{D} \frac{\rho}{2} U_{m}^{2}
$$

where $D$ denotes the pipe diameter, $U_{m}$ is the mean flow velocity and $L$ the length of the fluid portion.

For very large Reynolds numbers, the velocity distribution in the near wall fully turbulent region is given by

$$
\frac{u}{u_{*}}=\frac{1}{\varkappa} \ln \left(\frac{y u_{*}}{v}\right)+A,
$$

where $y$ is the wall distance, $u_{*}$ is the friction velocity $\left(=\sqrt{\tau_{w} / \rho}\right), \varkappa(=0.4)$ is von Karman's constant and $A=5.5$ (Schlichting [16]).

The integration of Eq. (2) over the cross-sectional area of a pipe shows that the universal law of friction for a smooth pipe should yield a straight line provided $f^{-0.5}$ is plotted against $\log \left(R_{e} f^{0.5}\right)$. Validation against the data of different experiments (Schlichting [16]) shows that

with $R_{e}=U_{m} D / \nu$.

$$
\frac{1}{\sqrt{f}}=2.0 \log \left(R_{e} \sqrt{f}\right)-0.8
$$

\subsection{Resistance law for single phase flow in smooth pipes with wall transpiration}

For flow subject to wall transpiration, Eq. (3) is clearly ineffective. One effect of fluid transpiration in pipe flow is to distort the velocity distribution throughout the pipe diameter so that friction drag is either reduced or increased. Thus, any advanced equation for the estimation of the friction coefficient should explicitly incorporate the transpiration rate in addition to the bulk velocity.

In Silva Freire [15], the matched asymptotic expansions method was applied to the equations of motion to find a law of the wall with a bi-logarithmic term. Comparison with some experimental data showed the additive parameter $A$ to vary with the transpiration rate according to

$$
u^{+}=\frac{1}{\varkappa} \ln \left(y^{+}\right)+A+\frac{\Pi}{\varkappa} W\left(\frac{y}{\delta}\right)+v_{w}^{+}\left(\frac{1}{2 \varkappa} \ln \left(y^{+}\right)+\frac{A}{2}\right)^{2}+\frac{\tilde{\Pi}}{\varkappa} W\left(\frac{y}{\delta}\right),
$$

with $u^{+}=u / u_{*}, y^{+}=y u_{*} / v, v_{w}^{+}=v_{w} / u_{*}, v_{w}=$ normal velocity at the wall and

$$
A=5-512 \frac{v_{w}}{U}
$$

where $U$ is the maximum velocity at the pipe centerline and parameters $\Pi, \tilde{\Pi}$ and function $W$ are related to the universal wake function. 
The integration of Eq. (4) over the cross-sectional area of a pipe gives

$$
U_{m}=U-3.75 u_{*}-v_{w}\left(1.86 A+2.34 \ln \left(R e^{+2}\right)-5.47\right),
$$

with $R e^{+}=R u_{*} v^{-1}$.

Considering that

$$
\frac{U_{m}}{u_{*}}=\frac{2 \sqrt{2}}{\sqrt{f}},
$$

further algebraic manipulations yield

$$
\begin{aligned}
1= & \frac{\sqrt{f}}{2 \sqrt{2}}\left(2.5 \ln \left(R e^{+}\right)+A-3.75\right) \\
& +v_{w}^{++}\left(1.56 \ln ^{2}\left(R e^{+}\right)+(1.25 A-4.68) \ln \left(R e^{+}\right)+\frac{A^{2}}{4}+1.86 A+5.47\right),
\end{aligned}
$$

where

$$
v_{w}^{++}=\frac{v_{w}}{U_{m}} \quad \text { and } \quad R e^{+}=\frac{U_{m} D}{v} \frac{\sqrt{f}}{4 \sqrt{2}} .
$$

The transcendental equation, Eq. (8), gives $f$ for known values of $R e^{+}$and $v_{w}^{++}$.

\subsection{Resistance law for single phase flow in rough pipes}

For a rough pipe, Nikuradse [17] showed the law of resistance to reduce to

$$
\frac{u}{u_{*}}=\frac{1}{\varkappa} \ln \left(\frac{y}{k_{s}}\right)+B,
$$

where $k_{s}$ is a characteristic length of the roughness and $\mathrm{B}=8.5$ (completely rough regime).

In fact, $B$ is a function of $\operatorname{Re}_{k}\left(=k_{s} u_{*} v^{-1}\right)$. The behavior of $B$ for the three types of flow regime discussed in Nikuradse [17] has been largely discussed in literature.

Ligrani and Moffat [18] suggest

$$
B=8.5 \sigma+\frac{1-\sigma}{\varkappa} \ln \left(R e_{k}\right)+(1-\sigma) C,
$$

where $\operatorname{Re}_{k}=k_{s} u_{*} / \nu, C=5.1$ and $\sigma=\sin ((1 / 2) \pi g)$ with

$$
g=\frac{\ln \left(R e_{k} / R e_{k, s}\right)}{\ln \left(R e_{k, r} / R e_{k, s}\right)},
$$

$R e_{k, s}=5, R e_{k, r}=70$ and this approximation is valid in the range $5 \leq R e_{k} \leq 70$. For $R e_{k}$ $>R e_{k, r}, g=1$, whereas for $R e_{k}<R e_{k, s}, g=0$.

A resistance formula for flow in a rough pipe can then be obtained by integrating Eq. (10) over the cross-sectional area of a pipe. The result is

$$
f=\left(0.88 \ln \left(R / k_{s}\right)+0.35 B-1.33\right)^{-2} .
$$

A comparison of Eq. (13) with the experiments of Nikuradse shows that for a fully rough regime the logarithmic additive term should be replaced by 1.74 . 


\subsection{Resistance law for single phase flow in rough pipes with wall transpiration}

A law of resistance for rough pipes with wall transpiration can now be advanced provided the results of the previous sections are considered. Let us define $R e^{++}=R / k_{s}$ and $A_{k}=B$ $-512 v_{w} / U$. It follows immediately from Eqs. (8) and (13) that

$$
\begin{aligned}
1= & \frac{\sqrt{f}}{2 \sqrt{2}}\left(2.5 \ln \left(R e^{++}\right)+A_{k}-3.75\right)+v_{w}^{++}\left(1.56 \ln ^{2}\left(R e^{++}\right)\right. \\
& \left.+\left(1.25 A_{k}-4.68\right) \ln \left(R e^{++}\right)+\frac{A_{k}^{2}}{4}+1.86 A_{k}+5.47\right) .
\end{aligned}
$$

\subsection{Unit cell models}

The motion of large bubbles in a vertical liquid stream was studied by Dumitrescu [19], Davies and Taylor [20] and Nicklin et al. [21]. Wallis [22] discussed horizontal and vertical slug flows from the point of view of a unit cell. Compared to the simplicity of the original works, the model advanced by Dukler and Hubbard [3] was much more evolving. The model is based on the observation of a two-layered system. A fast moving slug overruns a slow moving film that is shed behind the upstream Taylor bubble and mixes with the surrounding fluid due to turbulence diffusion. Pressure drop is thus assigned to two effects: (i) the pressure drop that results from the acceleration of the slow moving liquid film to the full slug velocity and (ii) the pressure drop required to overcome the wall shear in the back section of the slug. The model predicts the unit cell velocity, slug velocity, film velocity, slug length, mixing eddy length and the length of the film region.

Recently, the unit cell model was given more simplified formulations. The work of Orell [4] considers a uniform film thickness (thus a cylindrical bubble) and disregards end effects so that the flow in the film region can be treated as stratified flow. The nose of the elongated bubble and the slug unit travel with a velocity that is specified through an experimental correlation.

In literature, the details of the D\&H model vary according to authors. In fact, some of the implementations are completely uncharacteristic to the original model, with additional hypotheses that often conflict with the considered modeling principles. In the following, both models are summarized so that no doubt remains regarding their implementation details.

\subsubsection{The model of Dukler and Hubbard}

The idealized unit cell slug model of Dukler and Hubbard [3] (Fig. 5) consists of a long bubble travelling over a liquid film that is followed by a liquid plug with a strong mixing region at the front. Since in the film region pressure is essentially constant, pressure losses
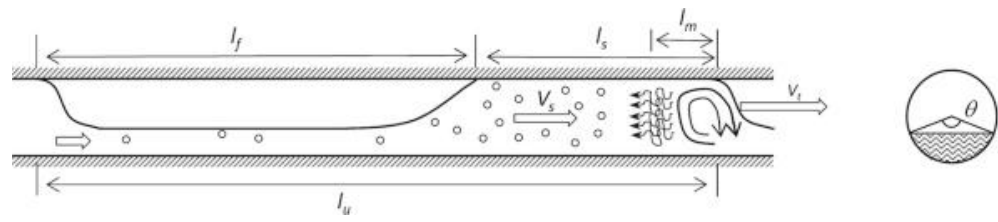

Fig. 5 The physical model of Dukler and Hubbard [3] 
are confined to the liquid slug region where they can be considered to result from the two effects described above: the acceleration of the slow moving liquid film to the unit cell velocity and the pressure loss required to overcome the wall shear stress in the main part of the liquid slug (Dukler and Hubbard [3]).

The mean velocity of the liquid slug $\left(V_{s}\right)$ is very close to the mixture velocity $\left(V_{m}\right)$,

$$
V_{s}=\frac{1}{A}\left(\frac{W_{L}}{\rho_{L}}+\frac{W_{G}}{\rho_{G}}\right),
$$

where $W$ is the mass flow rate and the subscripts $L$ and $G$ indicate the liquid and gas phases. $A$ is the area of the cross section of the pipe.

The effective Reynolds number in the liquid slug can be evaluated from

$$
R e_{s}=D V_{s} \frac{\rho_{L} R_{s}+\rho_{G}\left(1-R_{s}\right)}{\mu_{L} R_{s}+\mu_{G}\left(1-R_{s}\right)},
$$

where $R_{S}$ is the liquid holdup and $\rho$ stands for density.

The translational velocity of the unit cell is the mean velocity of the liquid slug added to the apparent velocity gained by fluid shedding from the preceding liquid slug. Parameter $C_{D H}$ expresses the ratio of the rate of shedding to the rate of flow in the liquid slug. A mass balance performed over a cross section of the flow in the liquid slug region can be used to estimate the shedding rate so that $C_{D H}$ can be expressed as

$$
C_{D H}=0.021 \ln R e_{s}+0.022 .
$$

There follows immediately that the translational velocity of the unit cell can be found from

$$
V_{t}=\left(1+C_{D H}\right) V_{s} .
$$

To find the pressure drop due to the acceleration of the film, the fractional area occupied by the film at the rear of the long bubble and the mean velocity at this position need to be known. The momentum and mass balances applied to the film region yield

$$
\int_{R_{f e}}^{R_{s}} W\left(R_{f}\right) d R_{f}=\frac{l_{f}}{D},
$$

and

$$
\frac{V_{t}}{v_{s}}-l_{f}=\frac{V_{s}}{v_{s}\left(R_{s}-R_{f e}\right)}\left[\frac{W_{L}}{\rho_{L} A V_{s}}-R_{f e}+C_{D H}\left(R_{s}-R_{f e}\right)\right] \text {, }
$$

where, $v_{s}$ is the frequency of passage of unit cells, $l_{f}$ the film length, $R_{f e}$ the liquid holdup of the film just before pick-up, and

$$
\begin{gathered}
W\left(R_{f}\right)=\frac{\frac{C_{D H}^{2} R_{s}^{2}}{R_{f}^{2}}-\frac{1}{F r}\left(\frac{\frac{\pi}{2} R_{f} \sin \frac{\theta}{2}+\sin ^{2} \frac{\theta}{2} \cos \frac{\theta}{2}}{1-\cos \theta}-\frac{1}{2} \cos \frac{\theta}{2}\right)}{f_{f} B^{2} \frac{\theta}{\pi}+\frac{R_{f}}{F r} \sin \beta}, \\
B=1-C_{D H}\left(\frac{R_{s}-R_{f}}{R_{f}}\right), \\
R_{f}=\frac{\theta-\sin \theta}{2 \pi}, \\
F r=\frac{V_{s}^{2}}{g D}, \\
R_{f}=\frac{2 \pi B R_{f}}{\theta} R_{s}, \\
f_{f}=0.0791 \operatorname{Re}_{f}^{-0.25} .
\end{gathered}
$$


The solution of Eqs. (19) and (20) furnishes $l_{f}$ and $R_{f e} . \theta$ is the angle that subtends the liquid film, as illustrated in Fig. 5b (see Dukler and Hubbard [3]).

The above equations can be rearranged to find $\theta_{f e}$ through an equation with the general form,

$$
\begin{gathered}
\mathcal{F}\left(\theta_{f e}\right)=\int_{\theta_{f e}}^{\theta_{S}} W^{\star}(\theta) d \theta-\mathcal{G}\left(\theta_{f e}\right)=0, \\
W^{\star}(\theta)=\frac{\frac{C_{D H}^{2} R_{s}^{2}}{\left(\frac{\theta-\sin \theta}{2 \pi}\right)^{2}}-\frac{1}{F r}\left(\frac{\frac{\pi}{2}\left(\frac{\theta-\sin \theta}{2 \pi}\right) \sin \frac{\theta}{2}+\sin ^{2} \frac{\theta}{2} \cos \frac{\theta}{2}}{1-\cos \theta}-\frac{1}{2} \cos \frac{\theta}{2}\right)}{0.0791\left(\frac{B(\theta-\sin \theta)}{\theta} R e_{s}\right)^{-0.25} B^{2} \frac{\theta}{\pi}+\frac{\left(\frac{\theta-\sin \theta}{2 \pi}\right)}{F r} \sin \beta} \\
\mathcal{G}\left(\theta_{f e}\right)=\frac{V_{s}}{v_{s} D\left[R_{s}-\left(\frac{\theta_{f e}-\sin \theta_{f e}}{2 \pi}\right)\right]} \\
\left\{\frac{W_{L}}{\rho_{L} A V_{s}}-\left(\frac{\theta_{f e}-\sin \theta_{f e}}{2 \pi}\right)+C_{D H}\left[R_{s}-\left(\frac{\theta_{f e}-\sin \theta_{f e}}{2 \pi}\right)\right]\right\}-\frac{V_{t}}{v_{s} D} .
\end{gathered}
$$

Provided $R_{f e}$ is known, parameter $V_{f e}$ can be determined from:

$$
V_{f e}=V_{s}\left[1-C_{D H}\left(\frac{R_{s}-R_{f e}}{R_{f e}}\right)\right] .
$$

The rate of liquid pick-up, the length of the liquid slug and the length of the mixing vortex region, can then be evaluated from

$$
\begin{gathered}
x=C_{D H} \rho_{L} A R_{f e}\left(V_{t}-V_{f e}\right), \\
l_{s}=\frac{V_{t}}{v_{s}}-l_{f}, \\
l_{m}=\frac{0.3\left(V_{s}-V_{f e}\right)^{2}}{2 g_{c}}\left(\frac{\rho_{L}}{s_{L}}\right),
\end{gathered}
$$

where $g_{c}$ is a conversion factor and $s_{L}$ is the specific weight of the liquid.

The total pressure loss is finally found from

$$
\Delta P=\Delta P_{a}+\Delta P_{f}
$$

where

$$
\begin{gathered}
\Delta P_{a}=\frac{x}{A}\left(V_{s}-V_{f e}\right), \\
\Delta P_{f}=\frac{2 f_{s}\left[\rho_{L} R_{s}+\rho_{G}\left(1-R_{s}\right)\right] V_{s}^{2}\left(l_{s}-l_{m}\right)}{D},
\end{gathered}
$$

and $f_{s}$ is the friction factor for the liquid slug.

\subsubsection{The model of Orell [4]}

The model of Orell [4] is based on the simplified model presented in Taitel and Barnea [23]. The model considers uniform film thickness and takes into account the increase of the apparent viscosity in the liquid slug due to the presence of air bubbles (Fig. 6). 


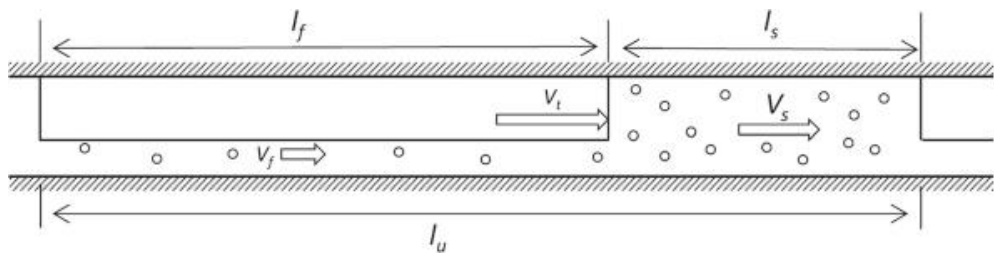

Fig. 6 The physical model of Orell [4]

The mass balance equations for the liquid and the gas phases are:

and

$$
V_{S L}=V_{s} R_{s} \frac{l_{s}}{l_{u}}+V_{f} R_{f} \frac{l_{f}}{l_{u}}
$$

$$
V_{S G}=V_{s}\left(1-R_{s}\right) \frac{l_{s}}{l_{u}}+V_{G}\left(1-R_{f}\right) \frac{l_{f}}{l_{u}},
$$

where $V_{G}$ is the velocity of the gas phase and $V_{s}$ is given by Eq. (15).

A liquid mass balance relative to a coordinate system that moves with the unit cell velocity furnishes

$$
\left(V_{t}-V_{f}\right) R_{f}=\left(V_{t}-V_{s}\right) R_{s},
$$

where $V_{t}$ is determined through the experimental expression advanced by Nicklin et al. [21]

$$
V_{t}=C V_{m}+V_{d},
$$

where $C=1.2, V_{m}$ is the mixture velocity and $V_{d}(=0.54 \sqrt{g D}$ for horizontal flow $)$ is the bubble drift velocity.

Combination of the momentum equation for both phases results in

$$
\frac{\tau_{f} S_{f}-\tau_{i} S_{i}}{A_{f}}=\frac{\tau_{G} S_{G}-\tau_{i} S_{i}}{A_{G}},
$$

where

$$
\begin{gathered}
\tau_{f}=\frac{1}{2} f_{f} \rho_{L} V_{f}^{2}, \\
\tau_{G}=\frac{1}{2} f_{G} \rho_{G} V_{G}^{2}, \\
\tau_{i}=\frac{1}{2} f_{i} \rho_{G}\left(V_{G}-V_{f}\right)^{2}, \\
f_{f}=\frac{C_{f}}{\left[\rho_{L} V_{f} D_{h f} / \mu_{L}\right]^{m}}, \\
f_{G}=\frac{C_{G}}{\left[\rho_{G} V_{G} D_{h G} / \mu_{G}\right]^{m}}, \\
f_{i}=0.0142,
\end{gathered}
$$

with $C_{f}=C_{G}=0.046$ and $m=0.2$ for turbulent flow. 
The liquid slug holdup is given by the correlation of Andreussi and Bendiksen [24], reformulated by Andreussi et al. [25]:

where

$$
R_{s}=1-\frac{V_{m} / \sqrt{g D}-F_{0}}{V_{m} / \sqrt{g D}+2400 B o^{-3 / 4}}
$$

$$
\begin{gathered}
B o=\frac{\left(\rho_{L}-\rho_{G}\right) g D^{2}}{\sigma}, \\
F_{0}= \begin{cases}2.6\left[1-2\left(\frac{D_{0}}{D}\right)^{2}\right], & \text { if } D \geq \sqrt{2} D_{0} \\
0, & \text { else }\end{cases}
\end{gathered}
$$

where $\sigma$ denotes the surface tension and $D_{0}=0.025 \mathrm{~m}$.

Equations (37) through (41) define a nonlinear system that needs to be solved for four variables: $\theta, V_{f}, V_{G}, l_{s} / l_{u}$. All four variables can be written in terms of $\theta$, so that just one transcendental equation needs to be solved numerically.

The pressure drop can be directly calculated as the sum of the losses in the liquid slug and liquid film:

$$
\frac{\Delta P}{l_{u}}=2 \frac{f_{s}}{D} \rho_{s} V_{s}^{2} \frac{l_{s}}{l_{u}}+\frac{\tau_{f} S_{f}-\tau_{i} S_{i}}{A_{f}} \frac{l_{f}}{l_{u}},
$$

where

$$
\begin{gathered}
\rho_{s}=\rho_{L} R_{s}+\rho_{G}\left(1-R_{s}\right), \\
\mu_{e f f}=\mu_{L}\left[1+2.5\left(1-R_{s}\right)\right], \\
\operatorname{Re}_{s}=\frac{\rho_{s} V_{s} D}{\mu_{e f f}}, \\
f_{s}=0.046 \operatorname{Re}_{s}^{-0.2} .
\end{gathered}
$$

The model evaluates the pressure gradient directly, without the need to specify the pressure drop per unit cell. As such, no information about frequency or slug length is necessary.

\subsection{The effects of wall transpiration on unit cell modeling}

Uniform mass injection through a pipe wall alters the pressure drop due to the acceleration of the injected fluid in the axial direction. The pressure drop resulting from wall shear is also affected through a dual effect: the fluid injection increases the mixture velocity of the flow but decreases the liquid friction coefficient.

In particular, the following flow parameters may be expected to increase: the slug translational velocity, the length of the unit cell, the frequency of slug passage and the acceleration pressure drop.

Since the length of the unit cell is unknown and the mixture velocity depends on the amount of liquid that is transpired through the wall, an iterative scheme needs to be used to find $V_{s}$ and all other flow properties, which now obviously change from cell to cell. Thus, in the extension of the model of Dukler and Hubbard to transpired walls, the addition of mass has to be considered in Eqs. (15) through (36). 
Equation (35) in particular needs to be modified to

$$
\Delta P_{a}=\frac{x}{A}\left(V_{s}-V_{f e}\right)+\frac{W_{w}}{A} V_{t},
$$

where $W_{w}$ is the wall mass flow rate.

The friction coefficient needs to be modified according to Eq. (14) due to its dependence on the injection rate and bulk velocity.

The changes in $f_{s}$ (Eq. (14)) as a function of the slug Reynolds number and the injection rate are shown in Fig. 7.

The model of Orell furnishes predictions of $\theta, V_{f}, V_{G}, l_{s} / l_{u}$ from which pressure losses can be inferred. Of course, with the fluid injection at the wall, $V_{S L}, V_{t}$ (through $V_{m}$ ) and $R_{S}$ vary from cell to cell. The friction coefficients, $f_{f}$ and $f_{s}$, vary with the local injection rate also according to Eq. (14).

The additional acceleration term to be considered in the model of Orell due to fluid injection at the wall is

$$
\frac{\Delta P}{l_{u}}=4 \rho_{L} v_{w} \frac{V_{t}}{D}
$$

An important input parameter in mechanistic slug flow models is the passage frequency of slugs, $v_{s}$. Several correlations can be found in literature, based on empirical and mechanistic reasonings. In the following, the correlation presented in Zabaras [26] was used. This author analized 339 data points to propose the following correlation

$$
v_{s}=0.0226\left(\frac{V_{S L}}{g D}\right)^{1.2}\left[\frac{19.75}{V_{m}}+V_{m}\right]^{1.2}\left[0.836+2.75 \sin ^{0.25}(\beta)\right] .
$$

The above expression is to be used in IS units and $\beta$ is the inclination of the pipe.

\section{Experiments}

Limited experimental works have been conducted for turbulent flow in porous pipes. The investigation of Olson and Eckert [27] described the effects of continuous mass injection

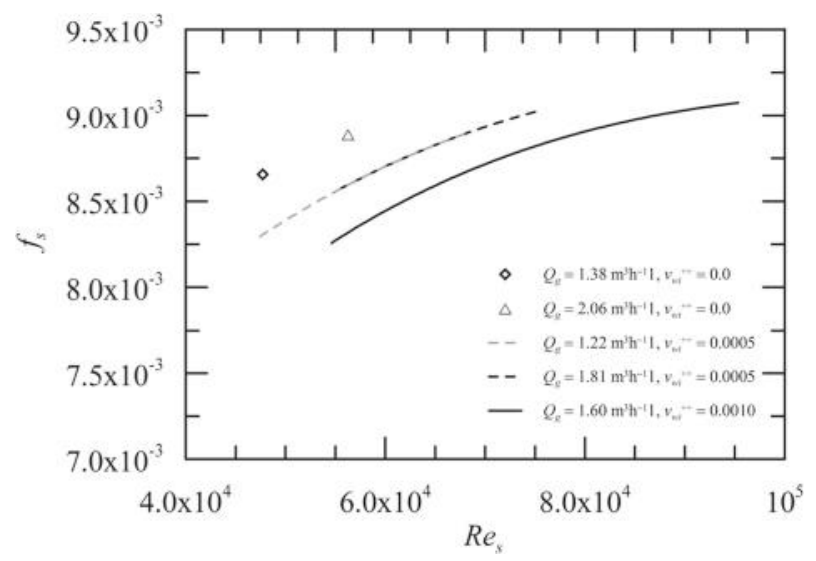

Fig. 7 Dependence of $f_{s}$ on $R e_{s}$ and $v_{w i}^{++}$ 
at the wall in the shape of the velocity profile and in the pressure loss. Results on friction factor and eddy diffusivity were also discussed. Su and Gudmundsson [28] and Ouyang et al. [29] provided further experimental data; the latter authors, in particular, considered also two-phase flows.

The present test section was assembled from 15 one-meter long units $(31.75 \mathrm{~mm}$ in diameter) consisting of three concentric stainless-steel tubes, as shown in Fig. 8. Water injected in the plenum chamber defined by the outermost circular ring migrates to the intermediate porous region and then onto the innermost producing pipe. Every one-meter long unit was fitted with two pressure taps and a dedicated magnetic flowrate meter. The units were connected together through a system of aligned flanges. Four plexiglass inspection windows were installed to allow for local velocity measurements and characterization of the twophase flow properties. Air and water were mixed in a T-junction located one meter upstream of the inlet of the porous pipe. A schematic diagram of the experimental setup is shown in Fig. 9.

Turbulent quantities were measured by a two-component Dantec Laser-Doppler Velocimetry System (LDV). The properties of the slug flow were measured through a Dantec Shadow Sizer System (SSS). Additionally, laser diodes and photo detectors were used to measure the properties of the two-phase flow along the pipe, so that changes in slug frequency, bubble velocity and lengths of the liquid slug and liquid film could be assessed.

The working principle of the phase detection sensor was very simple. A pair of diode lasers was aligned to a pair of photo detectors. Upon the passage of a large bubble, the laser beam was deflected by the interface, missing its target detector. An analog circuit was then used to condition the signal in a $0-8.5$ Volts range. The highest voltage corresponded to the presence of the liquid phase. An A/D converter together with further processing was used to define the train of bubbles.

For data reduction of the single phase model, the pressure losses due to flow acceleration needed to be accounted for. Pressure losses in a porous pipe with wall injection increase the momentum flux and overcome wall friction. A momentum balance on a control volume of length $d x$ and radius $R$ shows that the wall shear stress in given by

$$
\tau_{w}=-\frac{1}{4}\left[\frac{d p}{d(x / D)}+\frac{d\left(\beta \rho U_{m}^{2}\right)}{d(x / D)}\right],
$$

where $\beta$, the momentum flux factor, is the ratio of the real momentum flux through a given cross section to that based on one-dimensional flow at the mean velocity.
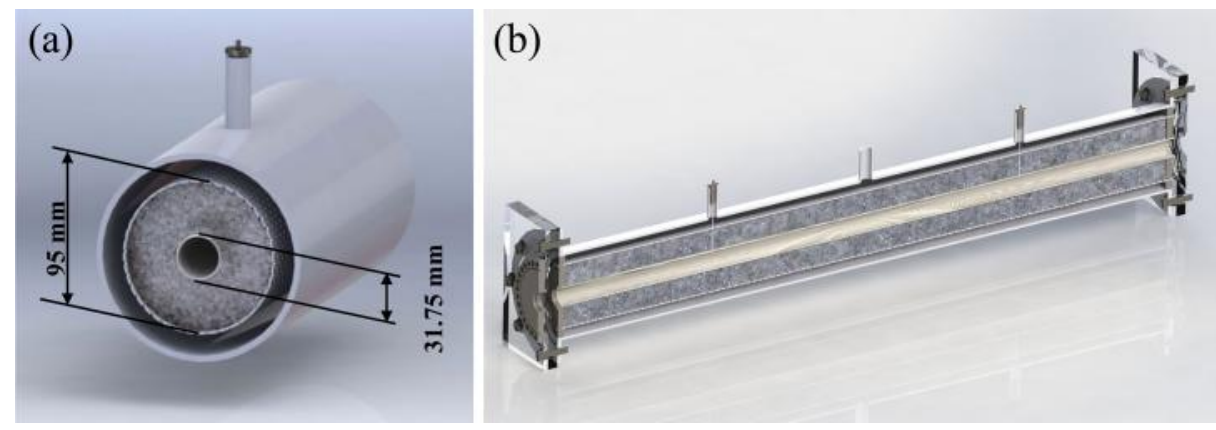

Fig. 8 Description of the test section: a) dimensions of the concentric pipes, b) detail of the one-meter long units 

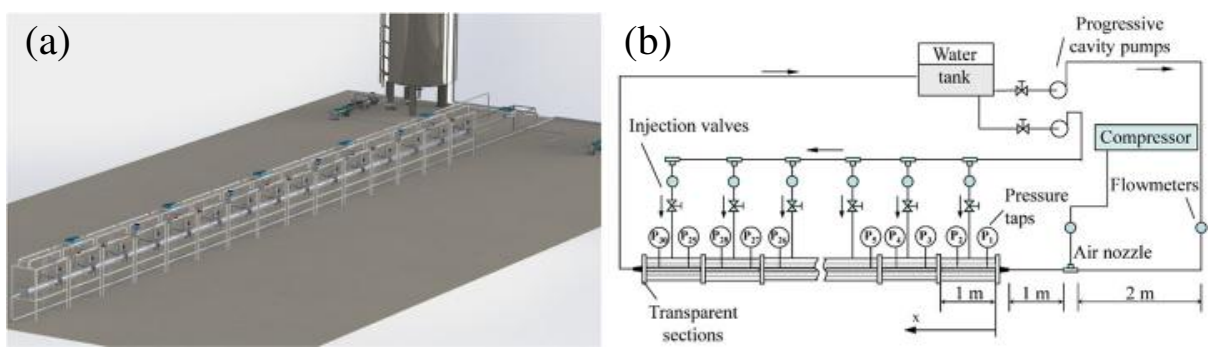

Fig. 9 Schematic diagram of the flow loop and test sections: (a) general view, (b) components of the experimental apparatus

For a full discussion on Eq. (59), please, refer to Olson and Eckert [27].

\section{Results and Discussion}

\subsection{Single-phase flow}

The roughness length $\left(k_{s}\right)$ of the test pipe was determined from single-phase water flow experiments with no injection at the wall. Various tests with Reynolds number varying from 6,000 to 100,000 were performed to find $k_{s}(=0.000334 \mathrm{~m})$.

Two distinct flow transpiration rates were studied to characterize the flow in the porous pipe, $v_{w i}^{++}=v_{w} / U_{m}=0.0005$ and 0.001 , where $U_{m}$ is the bulk velocity in the entrance section of the pipe. In the tests, the injection rate was kept constant in all of the fifteen one-meter long pipe segments.

The agreement provided by the predictions of Eq. (14) with the experimental data (Fig. 10) is very good despite the fact that the parameters in Eq. (14) were not particularly adjusted to fit the present experimental data. The values of the constants are the values presented in Loureiro and Silva Freire [2], and are based solely in the analysis of Silva Freire [15].

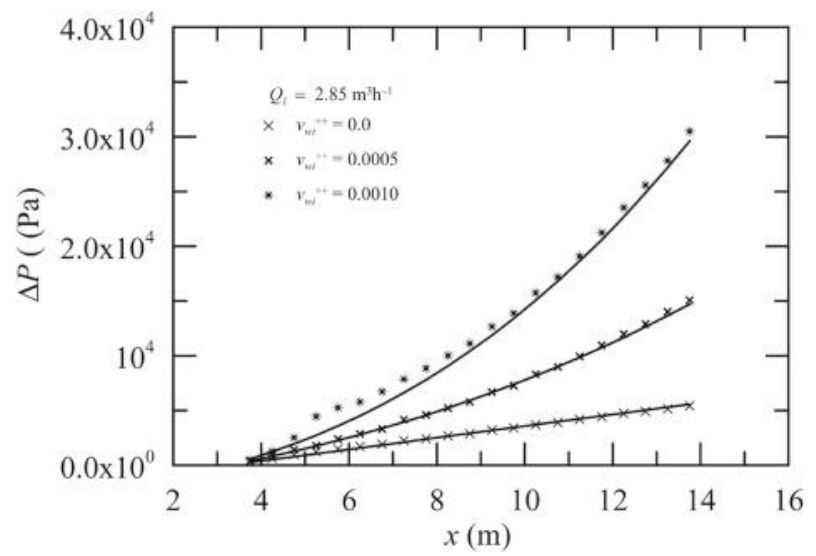

Fig. 10 Total pressure drop distributions for single-phase flow. Comparison between results for the unblown case and two different transpiration rates 


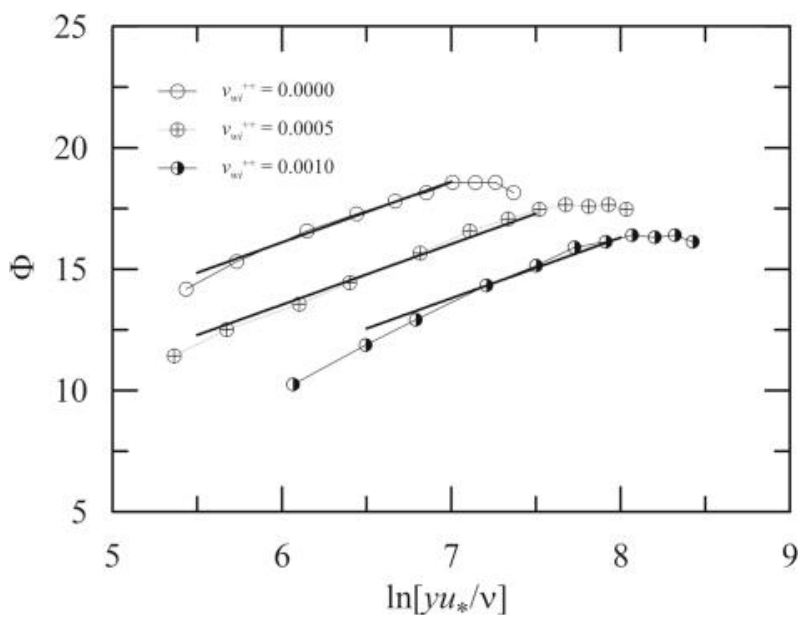

Fig. 11 Comparison between the results for the unblown case and two different transpiration rates studied: logarithmic mean velocity distributions

To investigate the logarithmic character of the velocity profile, Eq.(4) is re-written in the form

$$
\Phi=\frac{2}{v_{w}^{+}}\left[\left(v_{w}^{+} u^{+}+1\right)^{1 / 2}-1\right]=\frac{1}{\varkappa} \ln y^{+}+A .
$$

The mean velocity profile for all transpiration rates are shown in Fig. 11 according to the coordinates defined by Eq. (60). The existence of a bi-logarithmic region is plausible, with the level of the log-region decreasing as $v_{w}$ increases.

\subsection{Two-phase flow}

The two-phase flow experiments were conducted for the conditions shown in Table 1.

To characterize the influence of the transpiration flow rate on the slug flow behavior, the Shadow Sizer System was positioned at transparent windows located at positions $x=3,7$ and 13 metres downstream of the inlet section. A NanoSense MKIII camera provided high resolution images $(1289 \times 1024$ pixels $)$ at $2000 \mathrm{fps}$.

Table 1 Conditions of the multiphase flow experiments. $v_{w i}^{++}\left(=v_{w} U_{m}^{-1}\right)$ where $v_{w}$ is the normal injection velocity at the wall and $U_{m}$ is the bulk velocity in the inlet section of the pipe

\begin{tabular}{llll}
\hline Condition & $Q_{l}\left[\mathrm{~m}^{3} \mathrm{~h}^{-1}\right]$ & $Q_{g}\left[\mathrm{~m}^{3} \mathrm{~h}^{-1}\right]$ & $v_{w i}^{++}$ \\
\hline 1 & 2.46 & 1.38 & 0.0 \\
2 & 2.46 & 1.22 & 0.0005 \\
3 & 2.46 & 2.06 & 0.0 \\
4 & 2.46 & 1.81 & 0.0005 \\
5 & 2.46 & 1.60 & 0.0010 \\
6 & 2.85 & 1.45 & 0.0010 \\
7 & 2.85 & 1.90 & 0.0010 \\
8 & 2.85 & 3.59 & 0.0 \\
9 & 2.85 & 2.54 & 0.0010 \\
\hline
\end{tabular}



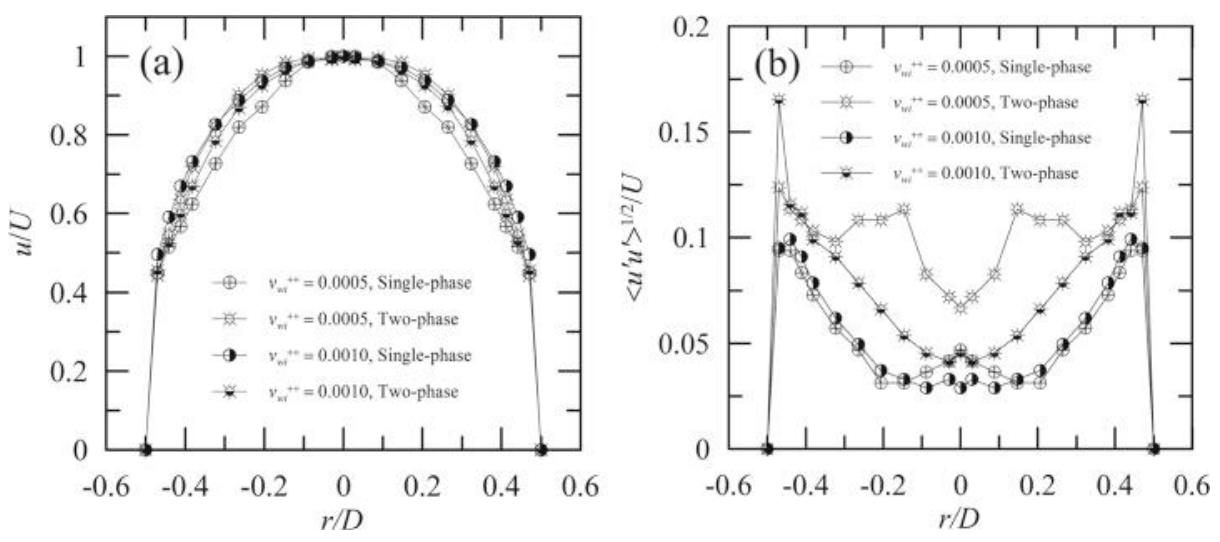

Fig. 12 Laser-Doppler anemometry measurements: (a) mean velocity profiles and (b) longitudinal velocity fluctuations for the single and two-phase flow conditions with and without flow transpiration. Profiles were normalized by pipe diameter $D$ and centerline velocity $U$. The mixture velocity at position $x=6 \mathrm{~m}$ was kept at $V_{m}=0.34 \mathrm{~ms}^{-1}$ for all test conditions

The local turbulent properties of the continuous phase in the liquid slug of transpired flows are discussed next. In particular, Fig. 12 illustrates the large degree of flow agitation provoked by the addition of mass at the wall. The flow conditions at the measuring station ( $x=6 \mathrm{~m}$ ) were adjusted so that the mixture velocity was kept at about $V_{m}=0.34 \mathrm{~ms}^{-1}$ for all test conditions. For both injection rates, high levels of turbulence are noticed throughout the cross section of the pipe for the two-phase flows. The phase interaction and the resulting bubble break up naturally induce the formation of turbulent wakes with the consequent general increase of turbulent intensity. Figure 12 shows that the increase in the streamwise velocity fluctuations can reach 2 to 3 times the levels found for single-phase flow. For the highest injection rate $\left(v_{w i}^{++}=0.001\right)$, a peak value of $0.17 U$ can be observed near the wall for $\sqrt{<u^{\prime} u^{\prime}>} / U$.

The changes in the lengths of the long bubbles as a result of the increase in turbulence level due to the wall injection are shown in Fig. 13. Measurements were carried out at

Fig. 13 Probability density function for the length of long bubbles in slug flow subject to wall injection

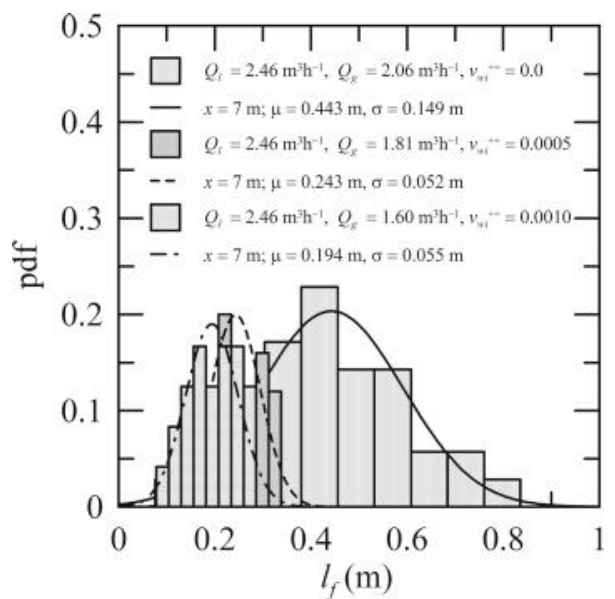


Fig. 14 Probability density function for the velocity of long bubbles in slug flow subject to wall injection

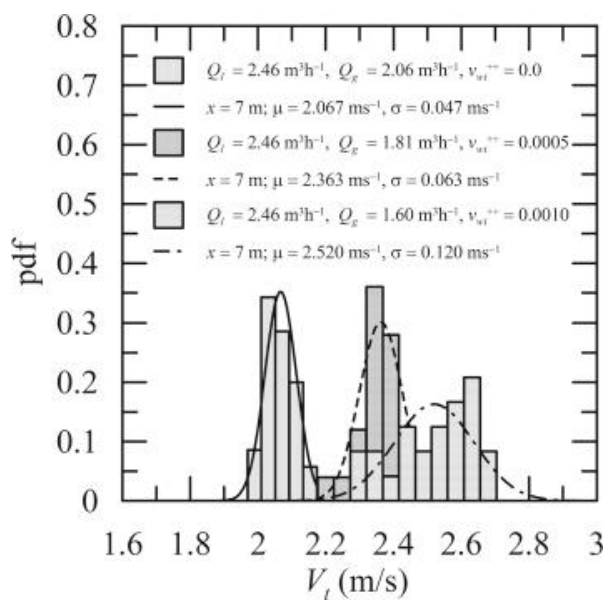

position $x=7 \mathrm{~m}$. For a fixed value of $Q_{l}\left(=2.6 \mathrm{~m}^{3} \mathrm{~h}^{-1}\right)$ and $Q_{g}$ about $2 \mathrm{~m}^{3} \mathrm{~h}^{-1}( \pm 0.4)$, the increase of $v_{w i}^{++}$largely reduces $l_{f}$. For zero injection rate, the average value of $l_{f}\left(\mu_{l f}\right)$ is $0.443 \mathrm{~m}$. With injections of $v_{w i}^{++}=0.0005$ and $0.001, \mu_{l f}$ is reduced to 0.243 and $0.194 \mathrm{~m}$ respectively.

The addition of mass at the wall impacts on the translational velocity of the large bubbles as shown in Fig. 14. The highest injection rate provokes an increase of $20 \%$ in $V_{t}$.

The effects of flow injection on the passage frequency of bubbles is shown in Fig. 15. The large increase in $v_{s}(50 \%)$ as $v_{w i}^{++}$varies from 0.0 to 0.001 , results possibly from the combined effects of the break up of the large bubbles and the increase in $V_{t}$.

Of course, the physical processes illustrated in Figs. 13 to 15 cannot be reproduced by the unit cell models previously discussed. However, it is of interest to study the results provided by the changes suggested in Subsection 3.6 on the prediction of slug flow properties.

The measured total pressure losses are compared with predictions obtained through the models of D\&H and Orell in Figs. 16 through 18.

The total pressure losses for flows without transpiration are shown in Fig. 16. For the lowest liquid and gas flow rates $\left(Q_{l}=2.46 \mathrm{~m}^{3} \mathrm{~h}^{-1} ; Q_{g}=1.38 \mathrm{~m}^{3} \mathrm{~h}^{-1}\right)$ the agreement between

Fig. 15 Probability density function for the passage frequency of long bubbles in slug flow subject to wall injection

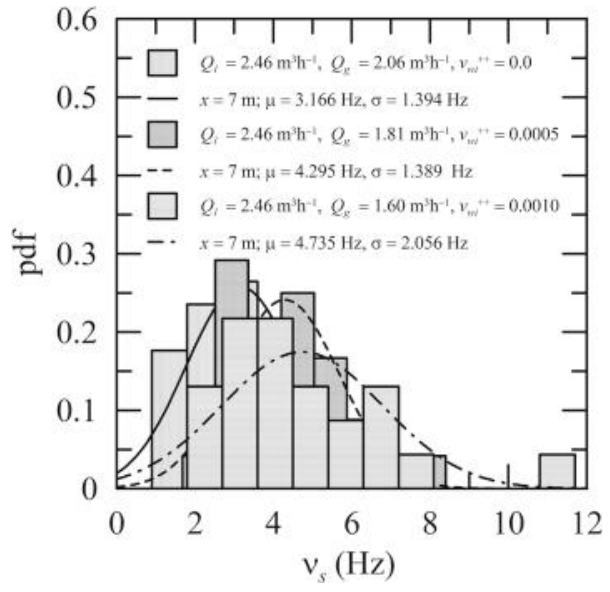




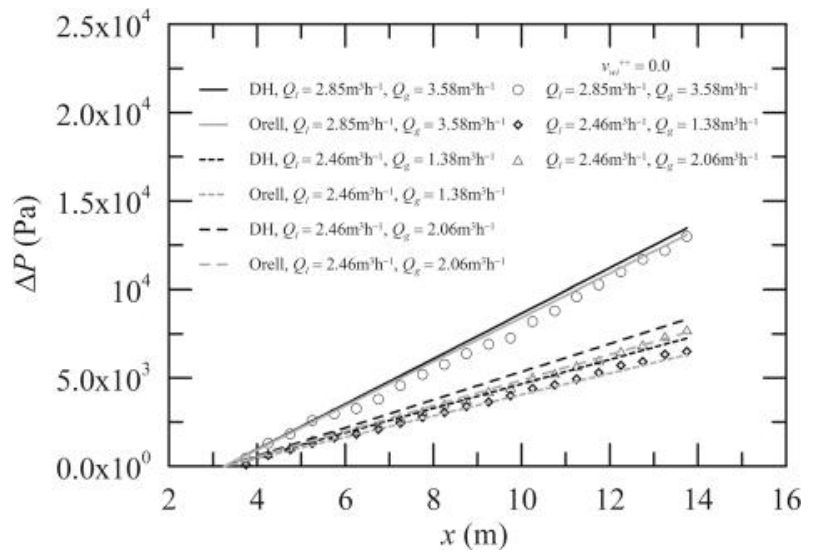

Fig. 16 Pressure drop predictions for two-phase flow without wall transpiration
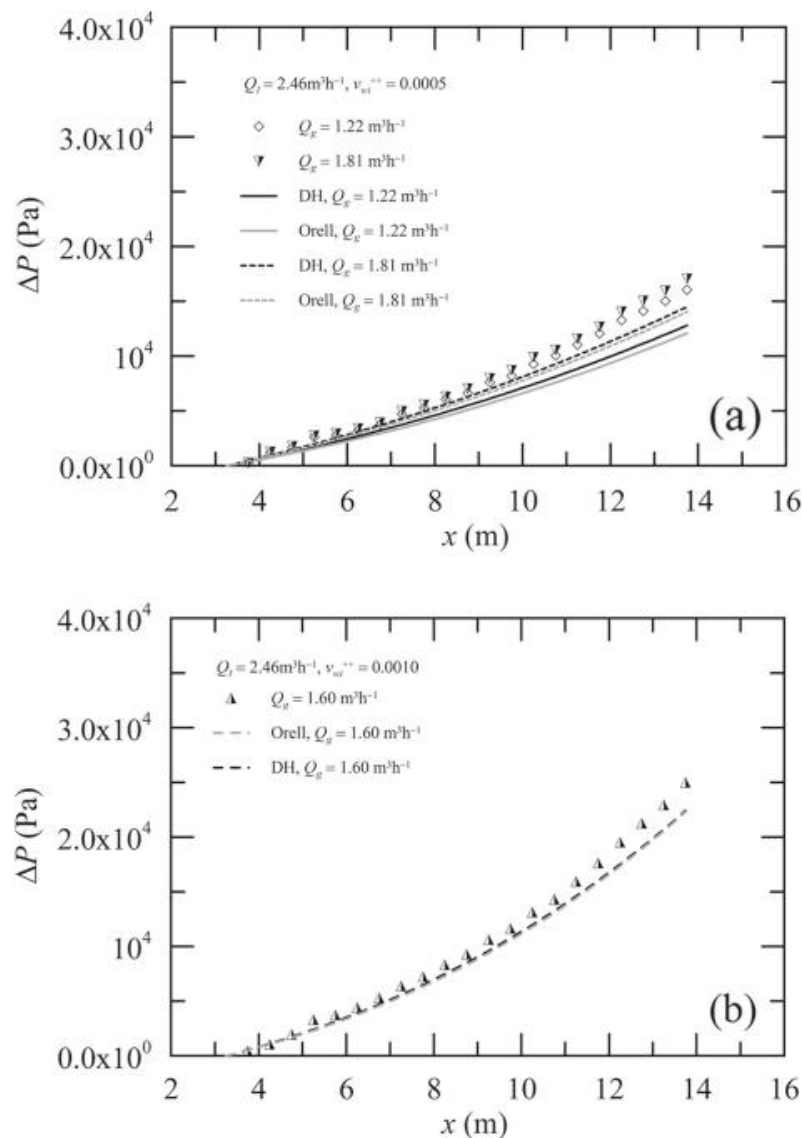

Fig. 17 Pressure drop predictions for two-phase flow with wall transpiration, $v_{w i}^{++}=0.0005$ (a) and 0.001 (b) 


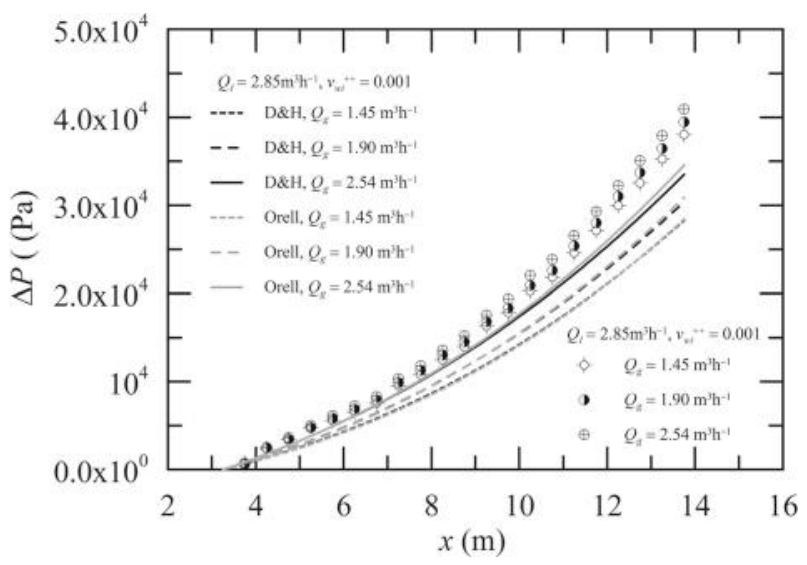

Fig. 18 Pressure drop predictions for two-phase flow with wall transpiration, $v_{w i}^{++}=0.001$

theories and experiments is within $11 \%(\mathrm{D} \& \mathrm{H})$ and $3 \%$ (Orell) in error. For the intermediate flow rates $\left(Q_{l}=2.46 \mathrm{~m}^{3} \mathrm{~h}^{-1} ; Q_{g}=2.06 \mathrm{~m}^{3} \mathrm{~h}^{-1}\right)$, the maximum differences reach $7 \%$ $(\mathrm{D} \& \mathrm{H})$ and $2 \%$ (Orell). For the highest flow rates $\left(Q_{l}=2.85 \mathrm{~m}^{3} \mathrm{~h}^{-1} ; Q_{g}=3.58 \mathrm{~m}^{3} \mathrm{~h}^{-1}\right)$, differences between predictions and experiments are below $3.5 \%(\mathrm{D} \& \mathrm{H})$ and $3 \%$ (Orell).

The increase in pressure drop in flows where fluid injection at the wall occurs is illustrated in Fig. 17a for the liquid flow rate $Q_{l}=2.46 \mathrm{~m}^{3} \mathrm{~h}^{-1}$ and injection rate $v_{w i}^{++}=0.0005$. For the two gas flow rates considered $\left(Q_{g}=1.22 \mathrm{~m}^{3} \mathrm{~h}^{-1}\right.$ and $\left.1.81 \mathrm{~m}^{3} \mathrm{~h}^{-1}\right)$ both modified models underpredict the experimental results. For the lowest $Q_{g}, \mathrm{D} \& \mathrm{H}$ and Orell underestimate the experimental data by $20 \%$ and $25 \%$ respectively. For the higher $Q_{g}$, the differences are within $15 \%(\mathrm{D} \& \mathrm{H})$ and $18 \%$ (Orell).

For the flow with $Q_{l}=2.46 \mathrm{~m}^{3} \mathrm{~h}^{-1}$ and injection rate $v_{w i}^{++}=0.001$, the models underpredict the pressure drop by $10 \%(\mathrm{D} \& \mathrm{H})$ and $11 \%$ (Orell) (Fig. 17b).
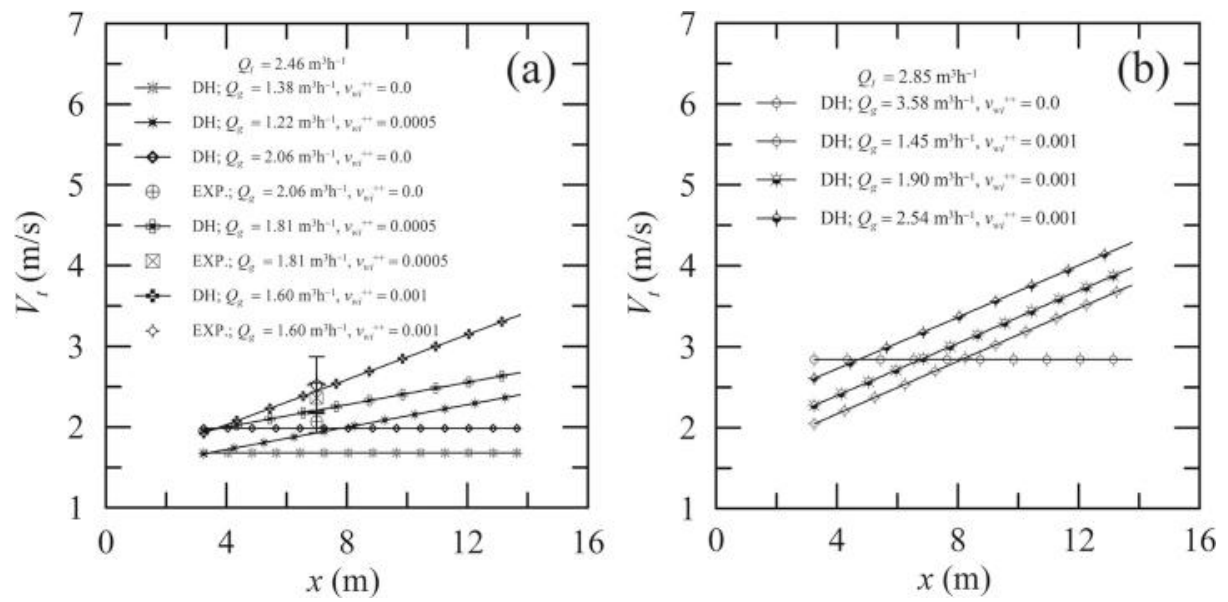

Fig. 19 Evolution of $V_{t}$ with flow injection at the wall according to the model of D\&H [3] 

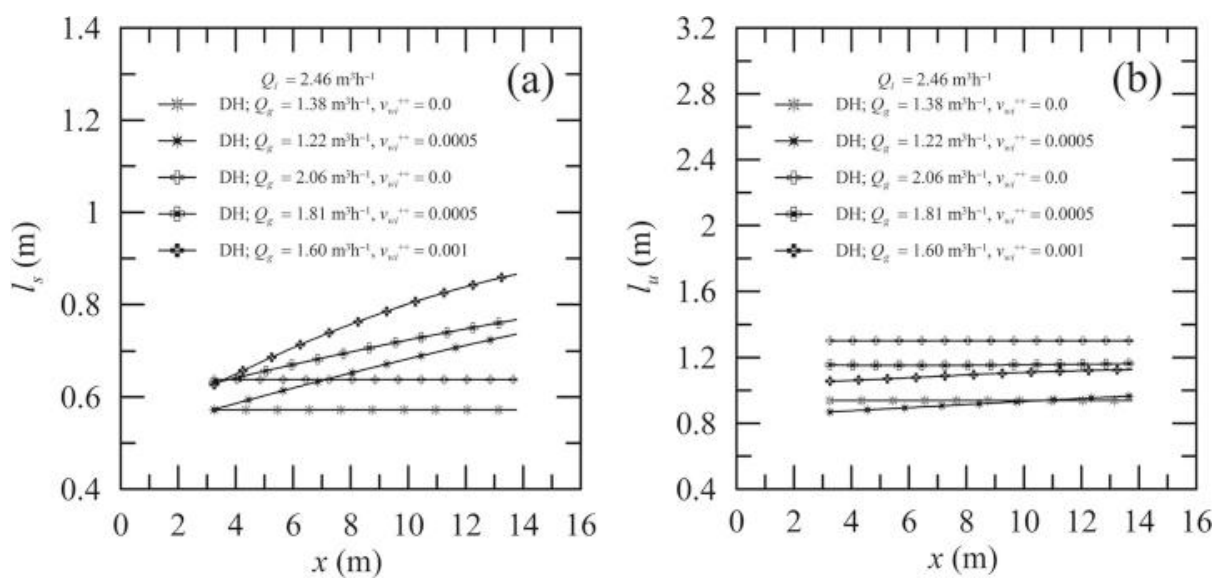

Fig. 20 Evolution of $l_{s}$ and $l_{u}$ with flow injection at the wall according to the model of D\&H [3]

To all of the above three experimental conditions, the predictions provided by the Orell model always gave lower results than those provided by the model of D\&H. However, it must be noticed that as $Q_{g}$ increases, results given by both models become very close.

For flows with $Q_{l}=2.85 \mathrm{~m}^{3} \mathrm{~h}^{-1}$ and injection rate $v_{w i}^{++}=0.001$, three different values of $Q_{g}$ were tested: $1.45,1.90$ and $2.54 \mathrm{~m}^{3} \mathrm{~h}^{-1}$ (Fig. 18). The distinction in predictions between the models of D\&H and Orell is very small. Both models underestimate the experimental data by $25 \%, 23 \%$ and $18 \%$ as $Q_{g}$ increases.

The effects of flow injection on $V_{t}$ are shown in Fig. 19a-b according to predictions of the extended D\&H model. The increase of the mixture velocity in the flow axial direction provokes an increase in $V_{t}$ for all flow conditions. Experimental results of $V_{t}$ are shown in Fig. 19a for $x=7 \mathrm{~m}$. The values of $V_{t}$ furnished by the D\&H model underpredict the measured data by $3 \%, 6 \%$ and $4 \%$ for $Q_{g}=1.60,1.81$ and $2.06 \mathrm{~m}^{3} \mathrm{~h}^{-1}$, respectively.

The overall behavior of $l_{s}$ and $l_{u}$ is shown in Fig. 20a-b. The wall addition of mass always increases $l_{s}$. The behavior of $l_{u}$ through the model of $\mathrm{D} \& \mathrm{H}$, however, varies with the gas flow rate. For the lowest gas flow rate $l_{u}$ increases with position, but for the highest flow rate $l_{u}$ does not vary significantly along the length of the pipe.

\section{Conclusions}

The present work has studied the behavior of single- and two-phase flows in horizontal pipes with fluid injection at the wall. Nine new experimental data sets are presented. In particular, the work discusses the mechanics of bubble break up by turbulence and the resulting effects on changes in the translational velocity, film length and the passage frequency of unit cells.

For single-phase flow, the results show that Eq. (14) furnishes pressure drop predictions that are good with an accuracy of about $3 \%$.

All two-phase flow experimental data are compared with extended versions of the theories of Dukler and Hubbard [3] and Orell [4]. Emphasis has been placed on the prediction of pressure distribution, but results for other properties including the translational velocity and characteristic lengths are discussed. The unit cell models are shown to furnish pressure distribution predictions about $20 \%$ below the experimental data. 
The corrections on the friction coefficient were observed to impact as much as $11 \%$ on predictions of the total pressure loss, although in average changes were of the order of $4 \%$. The acceleration corrections impacted the results in average on $12.5 \%$, with peak values of $14 \%$.

Acknowledgments In the course of the research, JBRL benefited from a CNPq Research Fellowship (Grant No 308445/2013-9) and from further financial support through Grants CNPq 458249/2014-9, FAPERJ E-26/102.212/2013 and FAPERJ E-26/010.002857/2014. APSF is grateful to the Brazilian National Research Council (CNPq) for the award of a Research Fellowship (Grant No 305338/2014-5). The work was financially supported by CNPq through Grant No 477293/2011-5 and by the Rio de Janeiro Research Foundation (FAPERJ) through Grant E-26/102.937/2011.

\section{References}

1. Townsend, A.A.: The structure of turbulent shear flow. Cambridge University Press (1976)

2. Loureiro, J.B.R., Silva Freire, A.P.: Slug flow in horizontal pipes with transpiration at the wall. Am. J. Phys. Conference Series (Online) 318 (2011)

3. Dukler, A.E., Hubbard, M.G.: A model for gas-liquid slug flow in horizontal and near horizontal tubes. Ind. Eng. Chem. Fund. 14, 337-347 (1975)

4. Orell, A.: Experimental validation of a simple model for gas-liquid slug flow in horizontal pipes. Chem. Eng. Sci. 60, 1371-1381 (2005)

5. Fabre, J., Liné, A.: Modeling of two-phase slug flow. Ann. R. Fluid Mech. 24, 21-46 (1992)

6. Matamoros, L.M.C., Loureiro, J.B.R., Silva Freire, A.P.: Length-area-volume of long bubbles in horizontal slug flow. Int. J. Multiphase Flow 65, 24-30 (2014)

7. Ujang, P.M., Lawrence, C.J., Hale, C.P., Hewitt, G.: Slug initiation and evolution in two-phase horizontal flow. Int. J. Multiphase Flow 32, 527-552 (2006)

8. Berman, A.S.: Laminar flow in channels with porous walls. J. App. Physics 24, 1232-1235 (1953)

9. Haldenwang, P.: Laminar flow in a two-dimensional plane channel with local pressure-dependent crossflow. J. Fluid Mech. 593, 463-473 (2007)

10. Chellam, S., Liu, M.: Effect of slip on existence, uniqueness, and behavior of similarity solutions for steady incompressible laminar flow in porous tubes and channels. Phys. Fluids 18, 083601 (2006)

11. Erdogan, M.E., Imrak, C.E.: On the axial flow of an incompressible viscous fluid in a pipe with a porous boundary. Acta Mech. 178, 187-197 (2005)

12. Tsangaris, S., Kondaxakis, D., Vlachakis, N.W.: Exact solution for flow in a porous pipe with unsteady wall suction and/or injection. Comm. Nonlinear Sci. Num. Simulation 12, 1181-1189 (2007)

13. Stevenson, T.N.: A Law of the wall for turbulent boundary layers with suction or injection. Cranfield Rep. Aero, vol. 166. The College of Aeronautics, Cranfield (1963)

14. Simpson, R.L.: The turbulent boundary layer on a porous plate: an experimental study of the fluid dynamics with injection and suction. Ph.D. Thesis. Stanford University, Stanford (1967)

15. Silva Freire, A.P.: An asymptotic solution for transpired incompressible turbulent boundary layers. Int. J. Heat Mass Transfer 31, 1011-1021 (1988)

16. Schlichting, H.: Boundary layer theory. McGraw Hill (1979)

17. Nikuradse, J.: Gesetzmässigkeiten der turbulenten strömung in glatten Rohren, Forschung ad Geb Ing, No 356 (1932)

18. Ligrani, P.M., Moffat, R.J.: Structure of transitionally rough and fully rough turbulent boundary layers. J. Fluid Mech. 162, 69-98 (1986)

19. Dumitrescu, D.T.: Stromung an einer luftblase im senkrechten Rohr. Z. angew. Math. Mech. 23, 139-149 (1943)

20. Davies, R.M., Taylor, G.I.: The mechanics of large bubbles rising through extended liquids and through liquids in tubes. Proc. Roy. Soc. 200A, 375-390 (1950)

21. Nicklin, D.J., Wilkes, J.O., Davidson, J.F.: Two-phase flow in vertical tubes. Trans. Inst. Chem. Eng. 40, 61-68 (1962)

22. Wallis, G.B.: One Dimensional Two-Phase Flow. McGraw-Hill (1969)

23. Taitel, Y., Barnea, D.: A consistent approach for calculating pressure drop in inclined slug flow. Chem. Eng. Sci. 45, 1199-1206 (1990) 
24. Andreussi, P., Bendiksen, K.: An investigation of void fraction in liquid slugs for horizontal and inclined gas-liquid pipe flow. Int. J. Multiphase Flow 15, 937-946 (1989)

25. Andreussi, P., Minervini, A., Paglianti, A.: Mechanistic model of slug flow in near-horizontal pipes. A.I.Ch.E. J. 39, 1281-1291 (1993)

26. Zabaras, G.B.: Prediction of slug frequency for Gas/Liquid flows. SPE J. 5, 252-258 (2000)

27. Olson, R., Eckert, E.: Experimental studies of turbulent flow in a porous circular tube with uniform fluid injection through the tube wall. J. Appl. Mech. 33, 7-17 (1966)

28. Su, Z., Gudmunsson, J.: Perforation inflow reduces frictional pressure loss in horizontal wellbores. J. Pet. Sci. Eng. 19, 223-232 (1998)

29. Ouyang, L.-B., Petalas, N., Arbabi, S., Schroeder, D.E., Aziz, K.: An Experimental Study of SinglePhase Fluid Flow in Horizontal Wells. SPE Western Regional Meeting, Bakersfield (1998) 